Journal of Medical Genetics 1989, 26, 545

\title{
The clinical significance of de novo structural rearrangements and markers detected prenatally by amniocentesis
}

Although amniocentesis and chromosome analysis of cultured amniotic fluid cells has been widely available for many years now, there are still occasions when the clinical significance of certain cytogenetic findings is not clear and there is difficulty in counselling the families because of lack of data.

Warburton ${ }^{1}$ presented frequency data from 76952 prenatal diagnoses in the USA of de novo structural rearrangements and markers and added other cases from Canada, Germany, and France to estimate outcome. Even from such a large group the number of anomalies was small and she concluded that further studies and improved follow up were required. In total, 66 rearrangements were reported (nine inversions, 16 Robertsonian translocations, and 41 reciprocal translocations) of which 14 were terminated. Of the nine inversions, one was terminated and had facial clefts and one child had structural defects and severe mental retardation, while seven were apparently normal; however, only one had follow up beyond the first year. Of the 16 Robertsonian translocations, two were terminated (one with a ventricular septal defect) and 14 were apparently normal at birth with only one being followed up beyond the first year. Of the 41 reciprocal translocations, 11 were terminated, two of whom had anomalies (one bilateral renal agenesis and one with 'soft' dysmorphic signs difficult to interpret in a fetus), while 30 were apparently normal at birth; however, only two were followed up into the second year of life. In summary, of the 66 de novo rearrangements there was only one known living abnormal child, but of the 51 other live births only four were followed up beyond the first year of life.

Jacobs $^{2}$ reviewed the outcome of de novo rearrangements detected in newborn studies but again the numbers were small. The four children with Robertsonian translocations were normal and only one of the 13 with other de novo rearrangements, who all appeared normal at birth, developed problems (myoclonic epilepsy and death at three and a half years).

Outcome data are small for de novo supernumerary marker chromosomes. Of 39 in Warburton's report, ${ }^{1}$ the outcome is unknown in six and abnormalities of various sorts were seen in six others at termination or birth.

Macgregor et al (p 590 of this issue) present outcome data on de novo translocations detected at amniocentesis in the west of Scotland over a 16 year period. Of the eight cases, one was terminated for anencephaly and the others were normal at follow up examination, their ages ranging from 1.3 to $10 \cdot 3$ years. The authors state that data from other centres are required for improved genetic counselling.

The Association of Clinical Cytogeneticists has recently established a working party on de novo structural rearrangements and markers detected prenatally by amniocentesis with the aim of combining experience from centres in the United Kingdom and collecting sufficient information to be helpful to families in decision making when a de novo event occurs.

In the first stage of the study organised by the working party, all cytogenetic laboratories ottering a prenatal diagnostic service will be asked to identify previous cases of de novo rearrangements and supernumerary markers. A control for each case will be selected by participating laboratories matched for approximate time of amniocentesis, for sex of fetus, for maternal age, and for primary indication for amniocentesis. The second stage of the study will be the clinical follow up. As comprehensive information as possible will be collected for those fetuses terminated. Ethical approval will be sought for study of the live births and matched controls. It is envisaged that the cases and controls will be examined by a clinical geneticist from the local regional centre, a detailed medical and developmental history taken, and an assessment of behaviour and development made by one of the adaptive behaviour scales. Some centres, or groups of centres, may wish to initiate research projects in association with the main study and proposals are invited. However, the main object of the working party is to collect sufficient numbers so that risk figures of abnormality can be derived for use in genetic counselling.

\section{Dian Donnal Department of Medical Genetics, St Mary's Hospital, Hathersage Road, Manchester M13 OJH.}

\footnotetext{
References

1 Warburton D. Outcome of cases of de novo structural rearrangements diagnosed at amniocentesis. Prenat Diagn 1984;4:69-70.

2 Jacobs PA. Mutation rates for structural chromosome rearrangements in man. Am J Hum Genet 1981;33:44-54.
} 